\title{
Force Characteristics of In Vivo Tissue-engineered Myocardial Constructs Using Varying Cell Seeding Densities
}

\author{
*Ravi Birla, †Vikas Dhawan, *Yen-Chih Huang, †Ian Lytle, $¥$ Khajohn Tiranathanagul, \\ and $†$ David Brown \\ *Section of Cardiac Surgery, †Section of Plastic Surgery, and $¥$ Section of Internal Medicine, University of Michigan, Ann \\ Arbor, MI, USA
}

\begin{abstract}
Experiments have been successfully performed culminating in functional, vascularized, three-dimensional cardiac muscle tissue. Past experience in tissue engineering has led us to the understanding that cell seeding density plays a critical role in the formation and function of both in vitro and in vivo engineered tissues. Therefore, to improve upon the mechanics of this model and to facilitate the formation of myocardial tissue with improved functional performance, we sought to optimize the seeding density of cardiomyocytes in these constructs. Neonatal cardiac myocytes were isolated from 2-day-old Fischer 344 rat hearts. Silicone chambers containing fibrin gel were seeded with varying numbers of cardiac cells $(1,5,10$, and 20 million). Control chambers were prepared using fibrin gel alone. All of the chambers were then implanted around the femoral vessels of isogenic rats. Six constructs per cell seeding density group were implanted. Histological and immunohistochemical evaluation was performed via hematoxylin and eosin, von Gieson, and $\alpha$-sarcomeric actin staining protocols. Linear contractile force measurements were obtained for each construct following 4 weeks of in vivo
\end{abstract}

implantation. After an implantation period of 4 weeks, the newly formed cardiac constructs contained within the chambers were harvested. The femoral vessels within the constructs were found to be patent in all cases. With direct electrical stimulation, the constructs were able to generate an average active force that varied depending on their seeding density. Constructs with seeding densities of 1,5 , 10 , and 20 million cells produced an average active force of $208,241,151$, and $108 \mu \mathrm{N}$, respectively. The control constructs did not generate any active force on electrical stimulation. This study demonstrates the in vivo survival, vascularization, organization, and function of transplanted myocardial cells. It is also apparent that cell seeding density plays a direct role in the force generation and mechanical properties of these engineered constructs. Among different groups using varying cell seeding densities, we found that the group with 5 million cells generated maximum active force. Key Words: Cardiac myocytes-Active force-Cell seeding density-Implantation-Angiogenesis - Tissue engineering.
Cardiac tissue engineering can play a potential therapeutic role in the treatment of patients suffering from a variety of cardiac disorders such as congestive heart failure (CHF), myocardial infarction, or congenital disorders. The number of CHF patients alone is on the rise, as is the cost related to their health care. Cardiac myocytes have a limited ability to

\footnotetext{
doi:10.1111/j.1525-1594.2008.00591.x
}

Received June 2007; revised November 2007.

Address correspondence and reprint requests to Dr. Ravi K. Birla, Section of Cardiac Surgery, University of Michigan, A510E MSRB I, 1150 West Medical Center Drive, Ann Arbor, MI 48109, USA.E-mail: rbirla@umich.edu regenerate. Attempts have been made to rejuvenate infracted myocardial tissue by injecting myogenic cells, replacing scarred myocardium with tissueengineered constructs, and to inject cells with genetically engineered DNA to constituently produce a therapeutic protein.

Several strategies have been utilized to engineer three-dimensional (3-D) cardiac tissue constructs. Temperature-sensitive surfaces have been used to assemble two-dimensional monolayers of cardiac myocytes, which can then be stacked together to form 3 -D cardiac muscle constructs (1-4). Synthetic scaffolds fabricated from polyglycolic acid (PGA) have been used to generate 3-D constructs by seeding them with neonatal cardiac myocytes (5-9). In 
addition, other researchers have investigated various biodegradable gel systems consisting of gelatin alginate and collagen (10-14).

Researchers in different fields of tissue engineering have tried to evaluate the role of cell seeding density so as to eventually optimize their functionality while developing various tissue constructs using scaffolds. Mauck et al. found that higher seeding densities of chondrocytes on agarose hydrogels resulted in greater than a twofold increase in material properties of resulting constructs (15). Kim et al. optimized cell seeding and culture protocols using biodegradable matrices of PGA fibers to engineer smooth muscle tissue (16). They demonstrated that an optimized experimental system can lead to the development of smooth muscle tissue with cellular and biochemical characteristics comparable to native smooth muscle. Dar et al. attempted to achieve 3-D high-density cardiac constructs with a uniform cell distribution and found that the percent cell yield in alginate scaffolds decreased at higher seeding densities (17).

Our previous in vitro experiments with selfassembled cardiac constructs indicated that higher density does not necessarily yield better contractile force (18). Also, in a previously described model of in vivo myocardial tissue engineering, we have shown that vascularization of these constructs allows significant improvement in their functional properties (19). In the present study, we aimed to improve the contractile function of these cardiac constructs by optimizing the cell seeding density. Such optimized constructs could effectively replace scarred myocardial tissue, and thus, restore function.

In our previous studies, we have developed a new model to bioengineer functional 3-D heart muscle in vivo (19). We isolated neonatal cardiac myocytes from 2- to 3-day-old Fischer 344 (F344) rat hearts and implanted silicone chambers containing fibrin gel primary cardiac cells around the femoral vessels in the groin area of adult rats. By 3 weeks, the chambers were found to be filled with newly formed "flaps" of tissue, supported by robust angiogenesis from the femoral artery (FA) and vein. Evaluation of the histologic and functional properties of these tissue-engineered myocardial constructs demonstrated the in vivo survival, vascularization, organization, and functionality of the transplanted myocardial cells. During our initial studies, we focused on model development and functional performance of tissue constructs. In the current studies, we focus on the role of initial cell seeding number on the functional performance of the tissue-engineered cardiac constructs.

\section{MATERIALS AND METHODS}

\section{Animal protocol}

Institutional approval for animal use was granted by the University Committee for the Use and Care of Animals in accordance with the "Guide for the Care and Use of Laboratory Animals" (NIH publication 86-23, 1986). All materials were purchased from Sigma (St. Louis, MO, USA) unless otherwise specified.

\section{Isolation of neonatal cardiac myocytes}

The hearts were cut into fine pieces and suspended in a dissociation solution (DS) which consisted of $0.32 \mathrm{mg} / \mathrm{mL}$ collagenase type II (Worthington Biochemical Corporation, Lakewood, NJ, USA) and $0.6 \mathrm{mg} / \mathrm{mL}$ pancreatin dissolved in a buffer consisting of $116 \mathrm{mM} \mathrm{NaCl}, 20 \mathrm{mM}$ HEPES, $1 \mathrm{mM} \mathrm{Na}_{2} \mathrm{HPO}_{4}$, $5.5 \mathrm{mM}$ glucose, $5.4 \mathrm{mM} \mathrm{KCl}$, and $0.8 \mathrm{mM} \mathrm{MgSO}_{4}$. The digestion of the cardiac tissue was carried out in an orbital shaker for $5 \mathrm{~min}$ at $37^{\circ} \mathrm{C}$. The supernatant was then replaced with fresh DS, and the digestion process was continued for an additional $30 \mathrm{~min}$. Following digestion, the supernatant was collected and mixed with $5 \mathrm{~mL}$ of horse serum (Invitrogen Corporation, Auckland, New Zealand) then centrifuged at $1500 \mathrm{rpm}$ for $5 \mathrm{~min}$. The cell pellet was then resuspended in $5 \mathrm{~mL}$ of horse serum. The digestion process was repeated an additional two to three times with the addition of fresh DS to the original undigested tissue. Cells from all the rounds of digestion were pooled, centrifuged, and then suspended in plating medium consisting of $335 \mathrm{~mL}$ Dulbecco's modified Eagle's medium (DMEM), 85 mL M199, $25 \mathrm{~mL}$ fetal bovine serum, $50 \mathrm{~mL}$ horse serum, and $5 \mathrm{~mL}$ antibiotic-antimycotic solution (Invitrogen Corporation). The total number of cells was determined using a hemocytometer. The process of tissue digestion does not separate the two main cell sources, namely myocardiocytes and fibroblasts. As a result, both cell types are present in the final suspension, but at a constant and uniform concentration.

\section{Preparation of the chambers for implantation}

Silicone tubing (Cole Parmer Instrument Company, Vernon Hills, IL, USA), $3.1 \mathrm{~mm}$ inner diameter and $4.7 \mathrm{~mm}$ outer diameter, and a wall thickness of $0.79 \mathrm{~mm}$ was cut to a length of $10 \mathrm{~mm}$, making an internal volume of $80 \mu \mathrm{L}$. A longitudinal slit was made down the length of each tube to allow it to be opened lengthwise for placement around the intact femoral vessels (Fig. 1). The previously isolated cardiac myocytes were suspended in fibrin gel $(2340 \mu \mathrm{L}$ of $3.5 \mathrm{mg} / \mathrm{mL}$ fibrinogen with $60 \mu \mathrm{L}$ of 

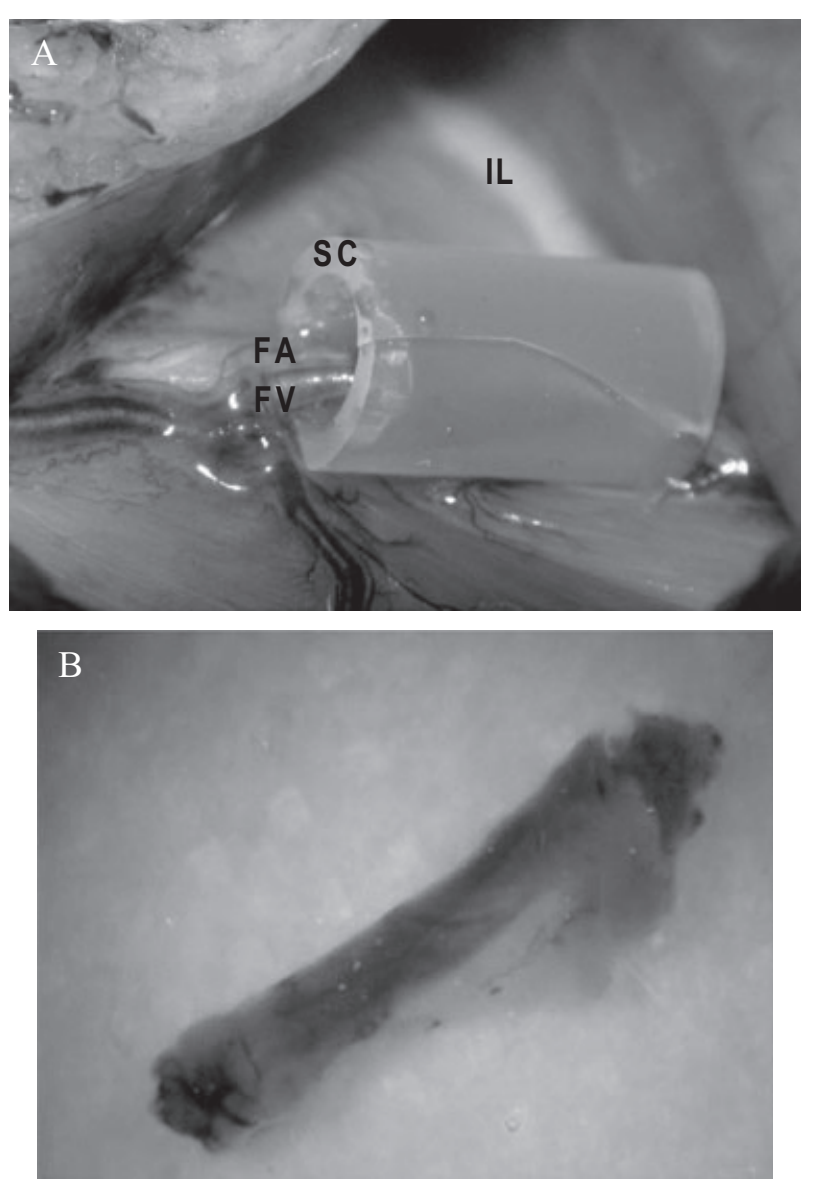

FIG. 1. Implantation of cardiac myocytes. (A) Implantation model: silicone chamber (SC) containing fibrin gel and cardiac myocytes implanted in the groin region around the FA, FV, and inguinal ligament (IL). (B) Cardiac construct after explantation.

$200 \mathrm{U} / \mathrm{mL}$ of thrombin) at a concentration of $1,5,10$, and 20 million cells (cardiomyocytes plus cardiofibroblasts) per $80 \mu \mathrm{L}$ of gel, respectively. The concentration of the cell suspension utilized was $12.5 \times 10^{6}$ and $62.5 \times 10^{6}$ cells $/ \mathrm{mL}$ for the 1 and 5 million group, respectively. For the 10 million group, we used a cell concentration of $1.25 \times 10^{8}$ cells $/ \mathrm{mL}$, and for the 20 million group we used $2.5 \times 10^{8}$ cells $/ \mathrm{mL}$. Eighty microliters of the cell and fibrin suspension was pipetted into vertically oriented silicone tubes (six chambers for each cell concentration) and allowed to gel. Additionally, six control chambers were prepared with fibrin gel alone.

\section{Implantation and retrieval of chambers}

Twenty-four cell-seeded chambers and six control chambers were implanted. Adult F344 rats, weighing approximately $250-300 \mathrm{~g}$, were used as recipients. The animals were anesthetized by an intraperitoneal injection of $0.5 \mathrm{~mL}$ of sodium pentobarbital (Abbott
Laboratories, Chicago, IL, USA). The groins were clipped of hair and sterilely prepped and draped. The femoral vessels were exposed through tangential incision made in the groin. With the aid of a dissecting microscope, the FA and femoral vein (FV) were separated from their surrounding tissue and accompanying femoral nerve. Several small vascular branches to the thigh musculature were cauterized to isolate the femoral vessels from the inguinal ligament to the branch point of the epigastric vessels. The previously prepared cardiomycoyte/fibrin gel-filled tubes were placed around the femoral vessels, by passing the intact vascular pedicle through the slit in the side of the silicone tubes (Fig. 1A). The wounds were closed in two layers, and the animals were allowed to recover.

After 4 weeks, the rats were reanesthetized and the chambers were isolated. Patency of the vascular pedicles was confirmed by visual observation of blood flow through the vessels. The FA, FV, and all soft tissue attachments were divided at the proximal and distal ends of the chambers. The silicone tubes were opened along their longitudinal slit, and the tissues within them were removed and pinned to $35 \mathrm{~mm}$ tissue culture plates coated with polydimethylsiloxane substrate (Dow Chemical Corporation, Midland, MI, USA). Culture medium consisting of $365 \mathrm{~mL}$ DMEM, $100 \mathrm{~mL}$ M199, $35 \mathrm{~mL}$ fetal bovine serum, and $5 \mathrm{~mL}$ antibiotic-antimycotic (Invitrogen Corporation) was added to the plates. The myocardial characteristics and contractility of the tissues were determined as described as follows.

\section{Functional assessment}

The functional capability of the cardiac construct was measured by active contractile force production. Immediately upon explantation, the constructs were placed in a $35 \mathrm{~mm}$ culture dish with DMEM growth medium. The method for evaluating the active contractile force of engineered muscle has been described in detail in previous work $(20,21)$. Briefly, the constructs were placed in culture media at $37^{\circ} \mathrm{C}$, between parallel platinum electrodes. One end of the construct was fixed to the plate by a pin and the other end of the construct was connected to a custom-built optical force transducer. The data I/O board was controlled by a custom-made LabVIEW software program (National Instruments, Austin, TX, USA) running on a personal computer. The LabVIEW and data I/O board generated output voltage signals which were amplified and delivered to the construct via platinum electrode wires lying parallel to the constructs, one electrode on either side of the construct. At the beginning of each contractile force testing 
session, the spontaneous contractions of the constructs were recorded in the absence of any electrical stimulation. This was followed by field stimulation to generate linear twitch contractile force. The following stimulation pulse parameters were utilized: a single bipolar, balanced, square wave pulse of $\pm 7 \mathrm{~V}$ amplitude and $1.2 \mathrm{~ms}$ duration.

\section{Assessment of cardiac muscle actin and collagen contents}

The cardiac muscle actin in the engineered cardiac tissue was visualized and evaluated by an immunofluorescence (IF) staining technique using antibodies raised against $\alpha$-sarcomeric actin. A standard collagen staining method using von Gieson stain and light microscopy was used to identify and evaluate the collagen content. Constructs were fixed in a $4 \%$ paraformaldehyde solution for $4 \mathrm{~h}$, rinsed in $70 \%$ ethanol, and stored in $70 \%$ ethanol until use. The constructs were prepared using a graded ethanol process with an automated tissue processor (Shandon Hypercenter XP, Thermo Electron, Waltham, MA, USA) for $7 \mathrm{~h}$ and $36 \mathrm{~min}$. The constructs were then paraffin embedded. The paraffinembedded samples were sectioned at $7 \mu \mathrm{m}$ on a microtome processor and placed on ProbeOn Plus slides (Fisher Scientific Company, Pittsburgh, PA, USA) for immunohistochemistry. For IF staining, the constructs were incubated with normal goat serum blocking solution for $30 \mathrm{~min}$, followed by mouse monoclonal anti- $\alpha$-sarcomeric actin (1:500, Sigma) primary antibody for $1 \mathrm{~h}$. Finally, they were incubated with goat FITC conjugated anti-mouse IgG (1:150, Sigma) for $30 \mathrm{~min}$. Consequently, the cardiac muscle actins were visualized in the tissue by using an IF microscope. Digital images were taken by an Olympus DP 70 digital camera (Center Valley, PA, USA) and associated software through an Olympus BX 51 microscope with the $10 \times$ objective lens. To demonstrate the whole tissue, a single image or two overlapping images were superimposed using Adobe Photoshop software (Adobe Systems, San Jose, CA, USA). Each whole tissue image was converted to a digital 8-bit, gray-scale image, and the brightness was adjusted until the mean value of negative tissue background equaled a predefined value of 25 (scale 0-255) using Adobe Photoshop software (Adobe Systems). This image was then analyzed using ImageJ software, version 1.34s (National Institutes of Health, Bethesda, MD, USA, download site http://rsb.info. nih.gov/ij/). First, the image was manipulated to remove background fluorescence via a threshold function. Only cardiac $\alpha$-sarcomeric actin that was fluorescent above the defined threshold level of 60 , on a 0-255 black-to-white scale, remained for analysis. The immunopositive region was measured and converted to an area in square millimeters. Next, the total tissue area was measured and the results were expressed as the percent area of fluorescently stained tissue to total tissue area.

Collagen fibers were identified by preparing the sectioned tissue with von Gieson stain. The digital images were taken in the bright field mode from the same microscope image system at the $4 \times$ magnification of the objective lens to demonstrate the whole tissue area. The photo was converted to a digital 8-bit, gray-scale image. The brightness was adjusted until the mean value of noncollagen background tissue equaled the predefined value of 120 (scale 0-255) using Adobe Photoshop software (Adobe Systems). This image was then analyzed using ImageJ software, version $1.34 \mathrm{~s}$ in a similar fashion stated earlier. In the case of collagen, the threshold value that represented positive staining of collagen fibers was defined as a value less than 100 (scale 0-255). The collagen content fraction was determined as the percent area of collagen to total tissue area.

\section{RESULTS}

After a period of 4 weeks of in vivo implantation, all 30 chambers were explanted. They were found to be filled with viable tissue occupying the entire volume of the chamber (Fig. 1B). The femoral vessels had incorporated into the newly formed tissue, and remained intact and patent. Each of the experimental constructs exhibited spontaneous contractions following explantation and placement in culture dishes filled with growth media at $37^{\circ} \mathrm{C}$. The stimulated contractile force of each of the explanted constructs was measured as per protocol. The twitch force of the explanted constructs varied with cell density (Fig. 2A), while control constructs did not generate any measureable active force in response to electrical stimulation (Fig. 2B). The average twitch force generated by the constructs with cell seeding densities of $1,5,10$, and 20 million cells was found to be 208,241 , 151, and $108 \mu \mathrm{N}$ (Fig. 3). Tissues harvested out of the control chamber on explantation did not exhibit any spontaneous contraction, and there was no active force generation on electrical stimulation.

Histological evaluation provided an insight into the cellular organization within the 3-D tissue constructs. Staining with hematoxylin and eosin $(\mathrm{H} \& \mathrm{E})$ revealed the presence of a large number of viable cells around the femoral vessels within the explanted chamber (Fig. 3). In addition, intact femoral vessels were observed within the 3-D 

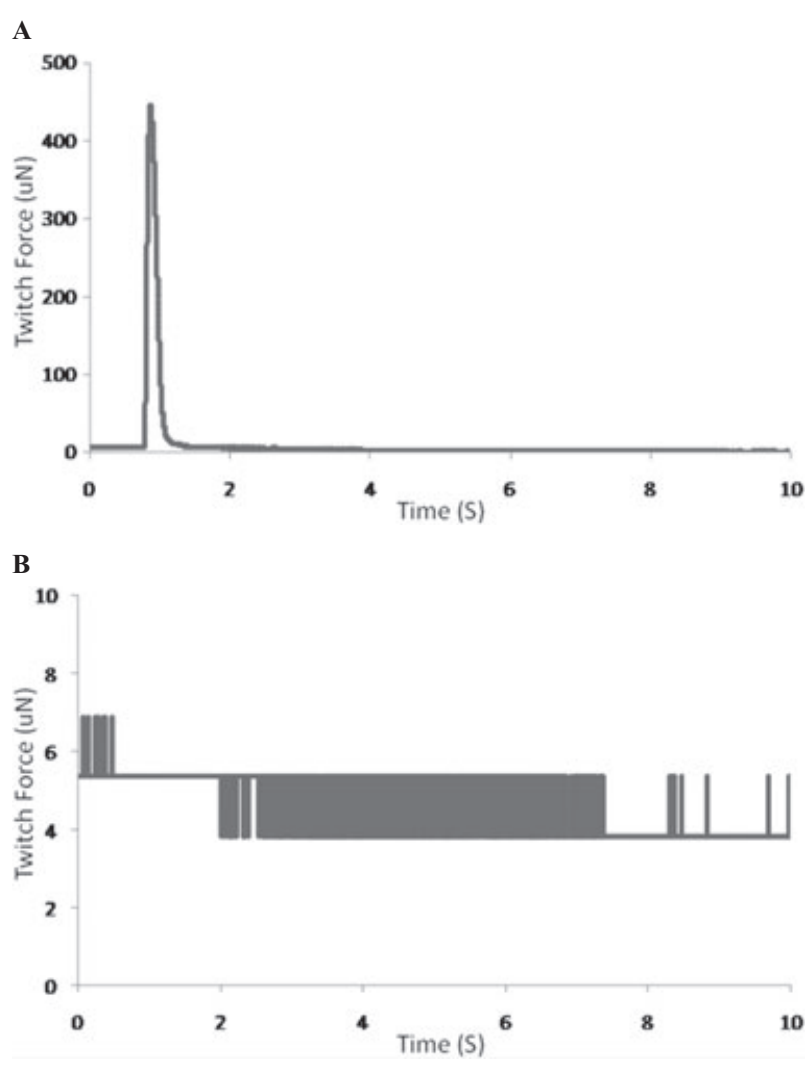

FIG. 2. Representative twitch force tracings are shown. Explanted cardiac constructs were stimulated between parallel platinum electrodes, and the twitch force was recorded using an optical force transducer. (A) Explanted cardiac construct with 5 million cells, and (B) control.

tissue construct. A large number of newly formed capillaries, with the presence of intraluminal erythrocytes, were also easily identifiable in the $H \& E$ stains of both the experimental and control constructs (Fig. 4). Staining for $\alpha$-sarcomeric actin showed the distribution of the cardiomyocytes. The cardiac cells were

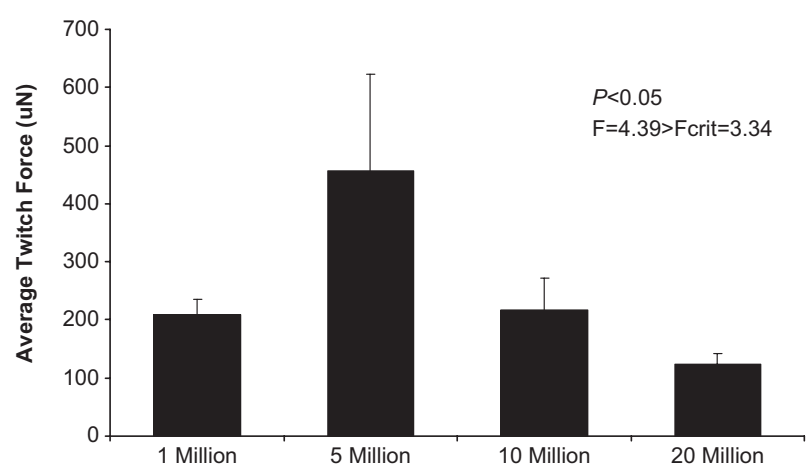

FIG. 3. In vivo cardiac construct active force. Graph showing the relationship between the cell seeding density and force generated by in vivo tissue-engineered myocardial constructs.


FIG. 4. Histology of the constructs. (A) H\&E staining of a section through the center of a construct demonstrates the presence of a large number of cells distributed throughout the tissue construct. (B) H\&E staining also shows the presence of vascular channels within the tissue construct.

predominantly distributed in a concentric ring of newly formed cellular material around the femoral vessels (Fig. 5). The percentages of $\alpha$-sarcomeric actin positive area per total construct tissue area with cell seeding densities of $1,5,10$, and 20 million cells were $13.97 \pm 2.73,18.88 \pm 1.43,16.95 \pm 1.11$, and $14.10 \pm 4.75 \%$ of total construct area, respectively ( $N=4$ and $P=0.59$ ) (Fig. 6). This finding demonstrates that the constructs with a cell seeding density of 5 million cells tend to have the highest percentage of actin, which corresponds with the active force data. There was no positive actin staining in the control constructs. There was also a significant amount of collagen present as identified by von Gieson staining. Large collagen fibers were concentrated closer to the femoral vessels. Smaller collagen fibers were found to be distributed throughout the concentric ring of cellular material. The percentages of collagen area per total construct tissue area with cell seeding densities of $1,5,10$, and 20 million cells were $21.45 \pm 4.03$, $14.67 \pm 1.02,15.37 \pm 3.29$, and $9.63 \pm 1.04 \%$ of total construct area, respectively $(N=4$ and $P=0.08)$ (Fig. 7).

\section{DISCUSSION}

Both cell transplantation and bioengineered cardiovascular tissues represent novel biologic 


\section{$H \& E-4 X$}

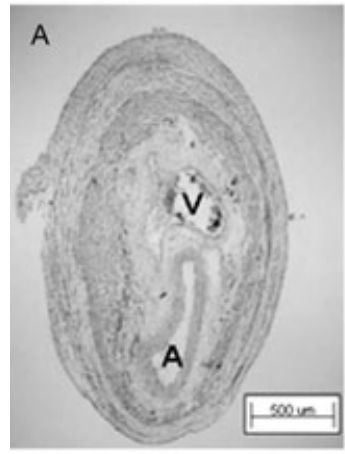

$40 \mathrm{X}$



von Gieson - 4X

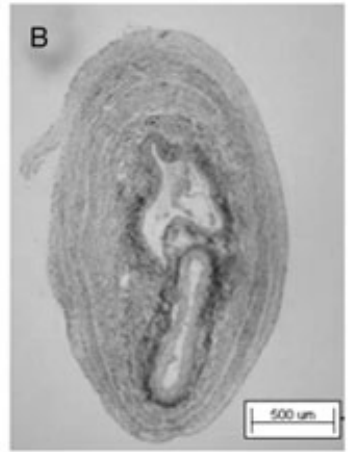

$40 \mathrm{X}$

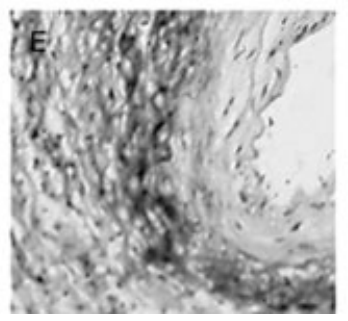

Actin - 4X

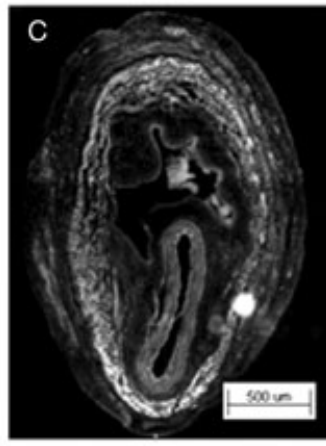

$40 \mathrm{X}$

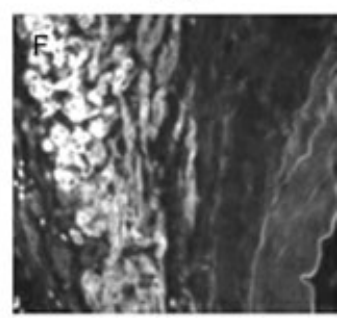

FIG. 5. Staining for collagen and actin. Serial cross sections of the cardiac construct stained in H\&E (A, D). A and V denoting the location of femoral artery $(A)$ and vein $(\mathrm{V})$ within the construct. von Gieson staining $(B, E)$ demonstrates the collagen fiber distribution. Predominant collagen presence is noted around the femoral vessels. Immunofluorescent $\alpha$-sarcomeric actin staining $(\mathrm{C}, \mathrm{F})$ demonstrating the concentric distribution of the cardiac cells around the femoral vessels within the construct. Histological sections presented here are for constructs generated with 5 million cells. approaches which can potentially benefit the patients with myocardial infarction, heart failure, and congenital heart diseases. The role of cell transplantation including seeding muscle cells such as cardiomyocytes or skeletal myoblasts as a means to repair damaged myocardium has been investigated. Seeding of noncardiac cells such as smooth muscle cells, endothelial cells, or bone marrow stem cells has been attempted to promote therapeutic angiogenesis and to induce differentiation of endogenous progenitors into myocytes in the infarct scar. Additionally, skeletal myocytes with genetically altered DNA have been produced which constituently manufacture proteins that stimulate angiogenesis. These cells have

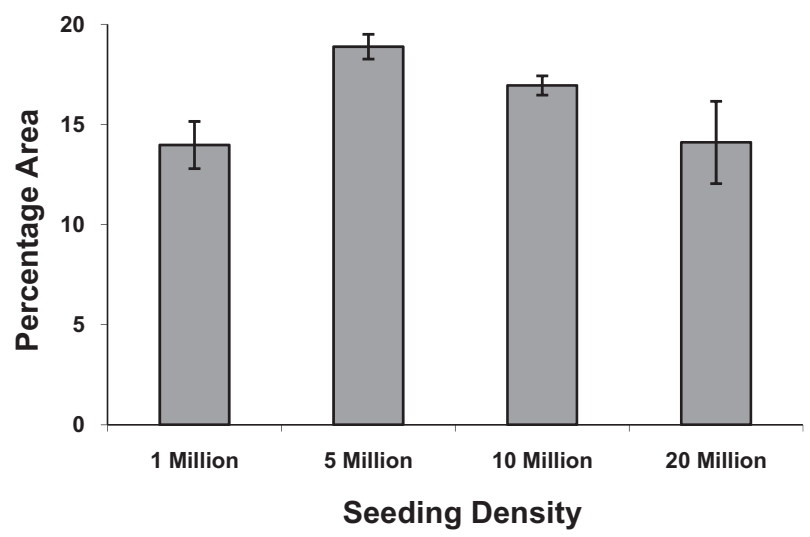

FIG. 6. Percentage area of $\alpha$-sarcomeric actin-stained cardiocyte content present in constructs with varying cell seeding densities. been injected into heart tissue and have delivered therapeutic proteins such as recombinant insulin-like growth factor-1 and recombinant vascular endothelial growth factor. Menasche et al. found that adult stem cells (myogenic or marrow derived) fail to electromechanically integrate within the recipient heart (22). They suggested that a true cardiomyogenic phenotype, such as cardiac stem cells or a cardiac precommitted embryonic stem cell, may have the potential to ensure a true regeneration of dead myocardium.

Elmadbouh et al. proposed the use of autologous mesothelial cells for cell therapy in the reversal of infarct scar modeling (23). Among bioengineered cardiovascular tissues, both scaffold-based and self-

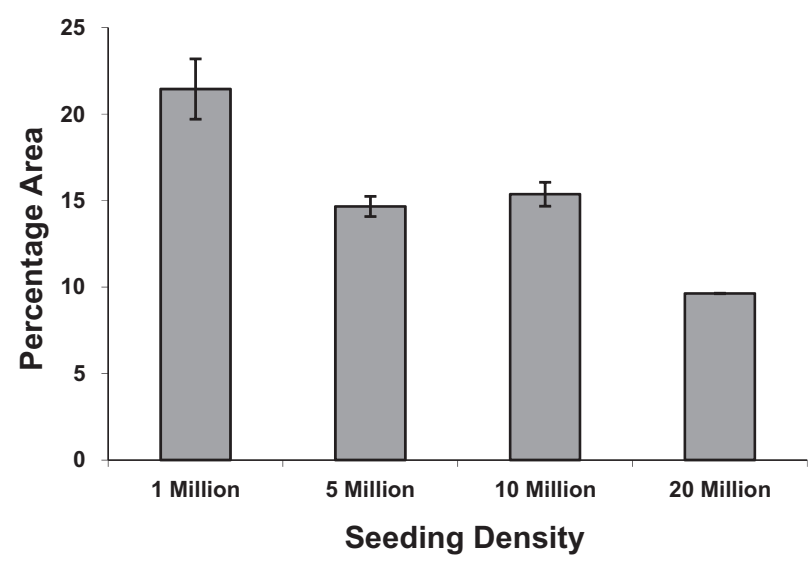

FIG. 7. Percentage area of collagen content present in constructs with varying cell seeding densities. 


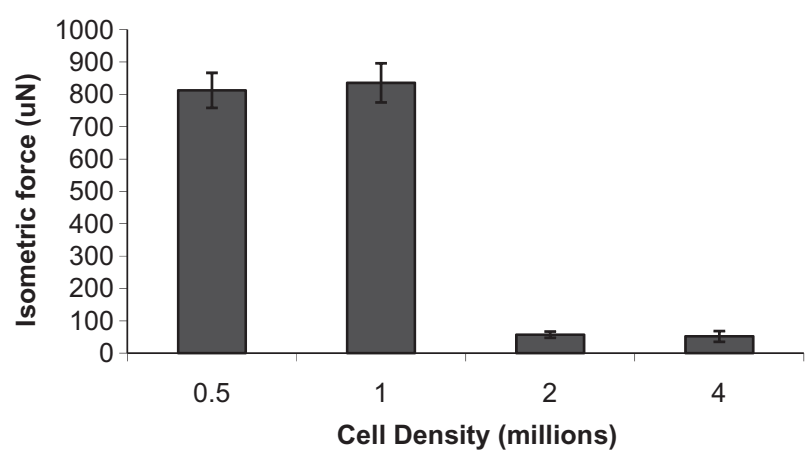

FIG. 8. Graph showing the relationship between the cell seeding density and force generated by in vitro tissue-engineered myocardial constructs.

assembled models of cardiac muscle constructs have been employed. In addition, both in vitro and in vivo techniques have been used to engineer these constructs. In our present work, we were able to reproduce our initial self-assembled model for in vivo cardiac tissue engineering. Additionally, we were able to study and understand the 3-D organization of cardiac cells within the in vivo formed constructs. We believe that factors such as cell seeding density, total implantation time, size of the chamber used, and fibrin degradation kinetics can influence the formation of these in vivo tissue-engineered constructs in terms of their ultimate functionality. In the present study, we aimed to find the optimal cell seeding density that would provide constructs with maximum contractile functionality. There have not been any published studies to evaluate the correlation between cell seeding density and resultant active force while forming self assembled in vivo tissue-engineered cardiac constructs.

Our initial in vitro experiments (18) suggested that higher cell seeding density does not result in a superior functioning construct (Fig. 8). The present in vivo study confirms these same results and demonstrates that the average active force generated by constructs with a seeding density of 5 million cells was higher as compared to constructs with seeding densities of 1 , 10 , or 20 million cells. Interestingly, quantification of positively stained actin also paralleled the force characteristics (Fig. 9). This suggests that the active force generated by these constructs is directly proportional to the quantity of viable cardiac tissue present within them. At low seeding densities of 1 million cells, there was less viable cardiac tissue as measured by actin content and less force produced. By increasing the seeding density to 5 million cells, the quantity of cardiac tissue, although not statistically significant, increased, as did force production. Once seeding

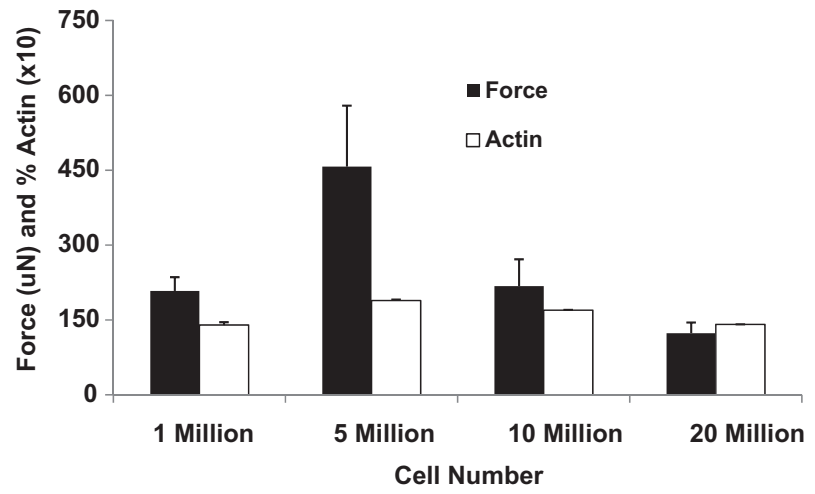

FIG. 9. Relationship between $\alpha$-sarcomeric actin-stained cardiac tissue and respective generated force in constructs with varying cell seeding densities. Values for percentage actin have been multiplied by a factor of 10 .

density was increased to greater than 5 million cells, cell density had a detrimental effect as seen by less force produced and decreased actin content in these constructs.

This suggests that although a high cell number was utilized for construct formation, there was an upper limit for cardiac cell viability. We speculate that a high degree of cell death was associated with the higher seeding density, thereby limiting the functional outcome of the explanted constructs. The collagen data also supported this notion. We found that the collagen content parallels both the force production and actin content at higher seeding densities. The collagen content decreased as the cell seeding density increased in the same manner as did the actin content at high seeding density. We speculate that this reduction of actin and collagen in the high-density seeded constructs may be due to cellular death of the transplanted cardiomyocytes and cardiofibroblasts secondary to the limited space available. Our results suggest that lower seeding densities of 5 million cells support the formation of more functional cardiac muscle due to high cardiac cell viability. Our study is, however, limited by the small sample size utilized. A total of six constructs were generated for each seeding density, and the constructs utilized for functional and histological testing. Although the results do provide a basis for the relationship between seeding density and functional performance of the tissue constructs, a larger sample size would have resulted in reduced bias of the data.

In summary, we have been able to optimize one of the important variables of our in vivo tissueengineered myocardial constructs, and demonstrate the effect of cell seeding density on resultant active force produced by them. We envision that 
tissue-engineered, 3-D constructs may have a potential role in the replacement and repair of infarcted myocardium.

Acknowledgment: The authors thank Dr. Quinghong Gao for assistance with collagen staining presented in this study.

\section{REFERENCES}

1. Shimizu T, Yamato M, Kikuchi A, Okano T. Two-dimensional manipulation of cardiac myocyte sheets utilizing temperatureresponsive culture dishes augments the pulsatile amplitude. Tissue Eng 2001;7:141-51.

2. Shimizu T, Yamato M, Akutsu T, et al. Electrically communicating three-dimensional cardiac tissue mimic fabricated by layered cultured cardiomyocyte sheets. J Biomed Mater Res 2002;60:110-7.

3. Shimizu T, Yamato M, Isoi Y, et al. Fabrication of pulsatile cardiac tissue grafts using a novel 3-dimensional cell sheet manipulation technique and temperature-responsive cell culture surfaces. Circ Res 2002;90:e40.

4. Shimizu T, Yamato M, Kikuchi A, Okano T. Cell sheet engineering for myocardial tissue reconstruction. [Review] [38 refs]. Biomaterials 2003;24:2309-16.

5. Bursac N, Papadaki M, Cohen RJ, et al. Cardiac muscle tissue engineering: toward an in vitro model for electrophysiological studies. Am J Physiol 1999;277:t-44.

6. Carrier RL, Papadaki M, Rupnick M, et al. Cardiac tissue engineering: cell seeding, cultivation parameters, and tissue construct characterization. Biotechnol Bioeng 1999;64:580-9.

7. Papadaki M, Bursac N, Langer R, Merok J, Vunjak-Novakovic $\mathrm{G}$, Freed LE. Tissue engineering of functional cardiac muscle: molecular, structural, and electrophysiological studies. Am J Physiol Heart Circ Physiol 2001;280:H168-78.

8. Papadaki M. Cardiac muscle tissue engineering. [Review] [13 refs]. IEEE Eng Med Biol 2003;22:153-4.

9. Radisic M, Euloth M, Yang L, Langer R, Freed LE, VunjakNovakovic G. High-density seeding of myocyte cells for cardiac tissue engineering. Biotechnol Bioeng 2003;82:403-14.

10. Eschenhagen T, Fink C, Remmers U, et al. Three-dimensional reconstitution of embryonic cardiomyocytes in a collagen matrix: a new heart muscle model system. FASEB $J$ 1997;11:683-94.

11. Eschenhagen T, Didie M, Munzel F, Schubert P, Schneiderbanger K, Zimmermann WH. 3D engineered heart tissue for replacement therapy. Basic Res Cardiol 2002;97:Suppl 1:I14652.

12. Zimmermann WH, Fink C, Kralisch D, Remmers U, Weil J, Eschenhagen T. Three-dimensional engineered heart tissue from neonatal rat cardiac myocytes. Biotechnol Bioeng 2000;68:106-14.

13. Zimmermann WH, Schneiderbanger K, Schubert P, et al. Tissue engineering of a differentiated cardiac muscle construct. [See comments.] Circ Res 2002;90:223-30.

14. Zimmermann WH, Eschenhagen T. Cardiac tissue engineering for replacement therapy. [Review] [79 refs]. Heart Fail Rev 2003;8:259-69.

15. Mauck RL, Wang CC, Oswald ES, Ateshian GA, Hung CT. The role of cell seeding density and nutrient supply for articular cartilage tissue engineering with deformational loading. Osteoarthritis Cartilage 2003;11:879-90.

16. Kim EJ, Li RK, Weisel RD, et al. Angiogenesis by endothelial cell transplantation. [See comment.] J Thorac Cardiovasc Surg 2001;122:963-71

17. Dar A, Shachar M, Leor J, Cohen S. Optimization of cardiac cell seeding and distribution in 3D porous alginate scaffolds. Biotechnol Bioeng 2002;80:305-12.

18. Huang YC, Khait L, Birla RK. Contractile three-dimensional bioengineered heart muscle for myocardial regeneration. J Biomed Mater Res 2007;80:719-31.

19. Birla RK, Borschel GH, Dennis RG, Brown DL. Myocardial engineering in vivo: formation and characterization of contractile, vascularized three-dimensional cardiac tissue. Tissue Eng 2005;11:803-13.

20. Dennis RG, Kosnik PE. Excitability and isometric contractile properties of mammalian skeletal muscle constructs engineered in vitro. In Vitro Cell Dev Biol Anim 2000;36:327-35.

21. Kosnik PE, Faulkner JA, Dennis RG. Functional development of engineered skeletal muscle from adult and neonatal rats. Tissue Eng 2001:7:573-84.

22. Menasché P, Hagège AA, Vilquin JT, et al. Autologous skeletal myoblast transplantation for severe postinfarction left ventricular dysfunction. J Am Coll Cardiol 2003;41:1078-83.

23. Elmadbouh I, Chen Y, Louedec L, et al. Mesothelial cell transplantation in the infarct scar induces neovascularization and improves heart function. Cardiovasc Res 2005;68:307-17. 